\title{
THE M31 PIXEL LENSING PLAN CAMPAIGN: MACHO LENSING AND SELF-LENSING SIGNALS
}

\author{
S. Calchi Novati ${ }^{1,2}$, V. Bozza ${ }^{2,3}$, I. Bruni ${ }^{4}$, M. Dall'Ora ${ }^{5}$, F. De Paolis ${ }^{6,7}$, M. DominiK $^{8,15}$, R. Gualandi $^{4}$, \\ G. Ingrosso ${ }^{6,7}$, Ph. Jetzer ${ }^{9}$, L. Mancini ${ }^{10,2}$, A. Nucita ${ }^{6,7}$, M. SAfonova ${ }^{11}$, G. Scarpetta ${ }^{1,2,3}$, M. Sereno ${ }^{12,13}$, \\ F. Strafella ${ }^{6,7}$, A. Subramaniam ${ }^{11}$, A. Gould ${ }^{14}$ \\ (PLAN COLLABORATION) \\ ${ }^{1}$ Istituto Internazionale per gli Alti Studi Scientifici (IIASS), Via Pellegrino 19, I-84019 Vietri Sul Mare (SA), Italy \\ ${ }^{2}$ Dipartimento di Fisica "E. R. Caianiello," Università di Salerno, Via Giovanni Paolo II 132, I-84084 Fisciano (SA), Italy \\ ${ }^{3}$ INFN, Sezione di Napoli, Via Cinthia 9, I-80126 Napoli, Italy \\ ${ }^{4}$ INAF, Osservatorio Astronomico di Bologna, Via Ranzani 1, I-40127 Bologna, Italy \\ ${ }^{5}$ INAF, Osservatorio Astronomico di Capodimonte, Salita Moiariello 16, I-80131 Napoli, Italy \\ ${ }^{6}$ Dipartimento di Matematica e Fisica “E. De Giorgi,” Università del Salento, CP 193, I-73100 Lecce, Italy \\ ${ }^{7}$ INFN, Sezione di Lecce, Via Arnesano, I-73100 Lecce, Italy \\ ${ }^{8}$ SUPA, University of St Andrews, School of Physics \& Astronomy, North Haugh, St Andrews, KY16 9SS, UK \\ ${ }^{9}$ Institute for Theoretical Physics, University of Zürich, Winterthurerstrasse 190, 8057 Zürich, Switzerland \\ ${ }^{10}$ Max Planck Institute for Astronomy, Königstuhl 17, D-69117 Heidelberg, Germany \\ ${ }^{11}$ Indian Institute of Astrophysics, Bangalore 560 034, India \\ ${ }^{12}$ Dipartimento di Scienza Applicata e Tecnologia, Politecnico di Torino, Corso Duca degli Abruzzi 24, I-10129 Torino, Italy \\ ${ }^{13}$ INFN, Sezione di Torino, Via Pietro Giuria 1, I-10125, Torino, Italy \\ ${ }^{14}$ Department of Astronomy, Ohio State University, 140 West 18th Avenue, Columbus, OH 43210, USA \\ Received 2013 November 6; accepted 2014 January 12; published 2014 February 20
}

\begin{abstract}
We present the final analysis of the observational campaign carried out by the PLAN (Pixel Lensing Andromeda) collaboration to detect a dark matter signal in form of MACHOs through the microlensing effect. The campaign consists of about 1 month/year observations carried out over 4 years (2007-2010) at the $1.5 \mathrm{~m}$ Cassini telescope in Loiano (Astronomical Observatory of BOLOGNA, OAB) plus 10 days of data taken in 2010 at the $2 \mathrm{~m}$ Himalayan Chandra Telescope monitoring the central part of M31 (two fields of about $13^{\prime} \times 12^{\prime} \cdot 6$ ). We establish a fully automated pipeline for the search and the characterization of microlensing flux variations. As a result, we detect three microlensing candidates. We evaluate the expected signal through a full Monte Carlo simulation of the experiment completed by an analysis of the detection efficiency of our pipeline. We consider both "self lensing" and "MACHO lensing" lens populations, given by M31 stars and dark matter halo MACHOs, in M31 and the Milky Way, respectively. The total number of events is consistent with the expected self-lensing rate. Specifically, we evaluate an expected signal of about two self-lensing events. As for MACHO lensing, for full $0.5\left(10^{-2}\right) M_{\odot}$ MACHO halos, our prediction is for about four (seven) events. The comparatively small number of expected MACHO versus self-lensing events, together with the small number statistics at our disposal, do not enable us to put strong constraints on that population. Rather, the hypothesis, suggested by a previous analysis, on the MACHO nature of OAB-07-N2, one of the microlensing candidates, translates into a sizeable lower limit for the halo mass fraction in form of the would-be MACHO population, $f$, of about $15 \%$ for $0.5 M_{\odot}$ MACHOs.
\end{abstract}

Key words: dark matter - galaxies: halos - galaxies: individual (M31, NGC 224) - Galaxy: halo - gravitational lensing: micro

Online-only material: color figures

\section{INTRODUCTION}

Gravitational microlensing (Roulet \& Mollerach 1997) is the tool of choice for the investigation of the dark matter content of galactic halos (Strigari 2013) in form of compact objects (MACHOs). Since the original paper of Paczyński (1986), observational campaigns have been undertaken to this purpose toward the Magellanic Clouds (Moniez 2010), as a probe of the Milky Way (MW) halo, and toward the nearby Andromeda galaxy, M31 (Calchi Novati 2010). Although there is agreement in excluding that MACHOs can fill up the dark matter halos, some tension remains based on the difficulty of fully disentangling the lensing signal from the known (stellar) population ("self lensing"), as opposed to the dark matter signal (MACHO lensing).

\footnotetext{
${ }^{15}$ Royal Society University Research Fellow.
}

The EROS (Tisserand et al. 2007) and more recently the OGLE collaboration (Wyrzykowski et al. 2009, 2010, 2011a, 2011b), from observations toward the Large and Small Magellanic Clouds (LMC and SMC), put rather robust upper limits (at a 95\% confidence limit; CL) on the halo mass fraction in the form of MACHOs, $f$, below $10 \%$ up to $1 M_{\odot}$ MACHOs (and down to below 5\% around $10^{-2} M_{\odot}$ objects). On the other hand, the MACHO collaboration had reported a MACHO signal toward the LMC of about $f \sim 20 \%$ within the mass range (0.1-1) $M_{\odot}$ (Alcock et al. 2000; Bennett 2005).

To address the reasons for this disagreement, the self-lensing scenario, originally discussed in Sahu (1994), Wu (1994), and Gould (1995), has thereafter been the object of several analyses (de Rujula et al. 1995; Aubourg et al. 1999; Salati et al. 1999; Alves \& Nelson 2000; Gyuk et al. 2000; Jetzer et al. 2002; Mancini et al. 2004; Griest \& Thomas 2005; Calchi Novati et al. 2006, 2009b; Calchi Novati \& Mancini 2011; Calchi Novati et al. 2013). Alternative hypotheses have also 
been discussed, in particular proposing non-standard models of the LMC/SMC that may somehow enhance the expected selflensing rate (Zaritsky \& Lin 1997; Zhao \& de Zeeuw 1998; Gould 1998; Evans \& Kerins 2000; Zaritsky 2004; Besla et al. 2013).

The main advantage of the line of sight toward M31 (Crotts 1992; Baillon et al. 1993; Jetzer 1994) is that, as an external galaxy, we can fully map its own dark matter halo (roughly, at parity of the MACHO mass function and halo fraction, one expects about two-thirds of the MACHO signal, if any, to belong to the M31 halo, with the rest belonging to the MW halo along that line of sight). Because of the large $(770 \mathrm{kpc})$ distance to the sources, we enter here the "pixel lensing" regime of microlensing (Gould 1996). In particular, among other specificities, we recall the further degeneracy in the lensing parameter space among the physical event duration, the Einstein time, $t_{\mathrm{E}}$, and the impact parameter, $u_{0}$, which makes reliable, in most cases, only a determination of the FWHM duration, $t_{\mathrm{FWHM}}$, (Gould 1996; Wozniak \& Paczynski 1997; Gondolo 1999; Alard 2001; Riffeser et al. 2006; Dominik 2009). Additionally, as further addressed below, it results that the ratio of the expected self lensing over the MACHO lensing rate is larger with respect to that expected toward the LMC/SMC (quantitatively, this depends on the field of view and on the assumed MACHO mass function) and this further complicates the physical interpretation of the data along this line of sight. Indeed, the analysis of the self-lensing signal appears to be at the origin of the disagreement between the POINT-AGAPE collaboration (Calchi Novati et al. 2005), who reported evidence for a MACHO signal (a different analysis of POINT-AGAPE is discussed in Belokurov et al. 2005) and the MEGA collaboration (de Jong et al. 2006; but see also de Jong et al. 2004) who concluded that their signal could be fully explained by the expected self-lensing rate (see also the further analyses in Ingrosso et al. 2006, 2007).

Following the difficulty of disentangling the MACHO and the self-lensing signals by considering the full sets of events, the detailed analysis of single events turn out to be very important. Interestingly, all the analyses of this kind presented up to now, concerning three distinct microlensing candidates toward M31, indicate that the lens should more likely be attributed to the MACHO lensing population (Aurière et al. 2001; Riffeser et al. 2008; Calchi Novati et al. 2010).

The more recently undertaken M31 pixel lensing PAndromeda survey (Lee et al. 2012), which is largely superior to previous surveys in terms of monitored field of view, baseline extension, and cadence (all essential issues to both enhance the expected rate and characterize the signal well) and out of which the detection of six new microlensing candidates out of a first analysis of their first year of observation has been reported, promises to mark an important step forward in this framework.

As the PLAN collaboration, we have undertaken a pixel lensing survey campaign toward M31 based at the Cassini telescope in Loiano (OAB). Following a first pilot season with 11 consecutive nights of observations in 2006 (Calchi Novati et al. 2007), which essentially probed the feasibility of the project, we then undertook a campaign that eventually lasted four years, 2007-2010. In 2010, we extended the monitoring to the $2 \mathrm{~m}$ Himalayan Chandra Telescope (HCT). The results of the 2007 campaign have been discussed in Calchi Novati et al. (2009a), with the presentation of a fully automated selection pipeline out of which we had selected two new microlensing candidates, which we called OAB-N1 and OAB-N2 (hereafter, OAB-07-N1 and $\mathrm{OAB}-07-\mathrm{N} 2$, respectively, with the additional indication of the year of detection). OAB-07-N2 is the object of further analysis, including that of the lens proper motion (also thanks to additional data kindly provided by the WeCAPP collaboration, Riffeser et al. 2001, 2003), presented in Calchi Novati et al. (2010).

In the present work, we present the final analysis of the PLAN survey including all four years of observations, both the OAB and HCT data. In particular, we present a third microlensing candidate, already presented in Lee et al. (2012), discuss the expected signal, both self lensing and MACHO lensing, and compare it with the observed rate. In Section 2.1, we present the observational data; in Section 2.2, we highlight the main steps of data reduction and our photometry procedure. In Section 2.3, we outline the method of our automated pipeline and present the results for the search of microlensing candidates; in Section 2.4, we discuss our analysis to establish the expected signal: a full Monte Carlo simulation of the experiment completed by an analysis of the detection efficiency of our pipeline. In Section 2.5, we present the expected signal and discuss the MACHO lensing versus self-lensing issue as compared with the observed rate; in Section 3, we present our conclusions.

\section{M31 PLAN PIXEL LENSING SURVEY}

\subsection{Observational Data}

Most data of our pixel lensing campaign have been collected at the $1.5 \mathrm{~m}$ Cassini telescope in Loiano, Osservatorio Astronomico di Bologna (http://www.bo.astro.it/loiano/), $785 \mathrm{~m}$ above sea level close to the city of Bologna (Italy). The photometric monitoring was carried out using the Bologna Faint Object Spectrograph and Camera equipped with a CCD EEV LN/ 1300-EB/1 back illuminated and Anti-Reflection (AR) coated, read out noise $3.1 \mathrm{e}^{-}$pixel $^{-1}$ and gain 2.22 $\mathrm{ADU}_{\text {pixel }}{ }^{-1}$, pixel scale of $0^{\prime \prime} .58$ pixel $^{-1}$, and with $1340 \times 1300$ pixels for a total field of view of $13^{\prime} \times 12^{\prime} \cdot 6$. We monitored two fields of view around the inner $\mathrm{M} 31$ region, centered at R.A. $=0^{\mathrm{h}} 42^{\mathrm{m}} 50^{\mathrm{s}}$ and decl. $=41^{\circ} 23^{\prime} 57^{\prime \prime}$ (north) and decl. $=41^{\circ} 08^{\prime} 23^{\prime \prime}$ (south), with axes parallel to the south-north and east-west directions, just leaving out the very inner M31 bulge region (Figure 1). The data have been collected in two broadband filters (similar to Cousins $R$ and $I$ ), with exposure times up to 10 minutes per frame depending on the filter and on the moon level. The standard data reduction has been carried out within the IRAF package (http://iraf.noao.edu/).

The typical microlensing events we expect to detect are relatively faint flux variations (with flux deviations at maximum magnification fainter than about $R \sim 20$ ) lasting up to a few days. Given the available experimental setup, these features fix our observational requirements. In particular, we need a long enough overall baseline with a suitable sampling and high signal-to-noise ratio ( $\mathrm{S} / \mathrm{N})$ data, namely we ask for full and consecutive nights of observations for an overall period up to about two months. The details of the sampling of the first pilot season (2006), whose data are however not further considered in the following, and for the following four-year campaign (2007-2010), are reported in Table 1. Overall, the average weather conditions (humidity, cloud coverage) did not turn out to be optimal for our purposes, with our sampling full of unwanted gaps (in particular, the consequence of the non-optimal sampling will be made apparent by the following discussion of the failed microlensing candidate in 2008 and the analysis of the 2010 data below). Indeed, the fraction of at least partially clear nights has remained around $60 \%$, with overall 114 at least partially 


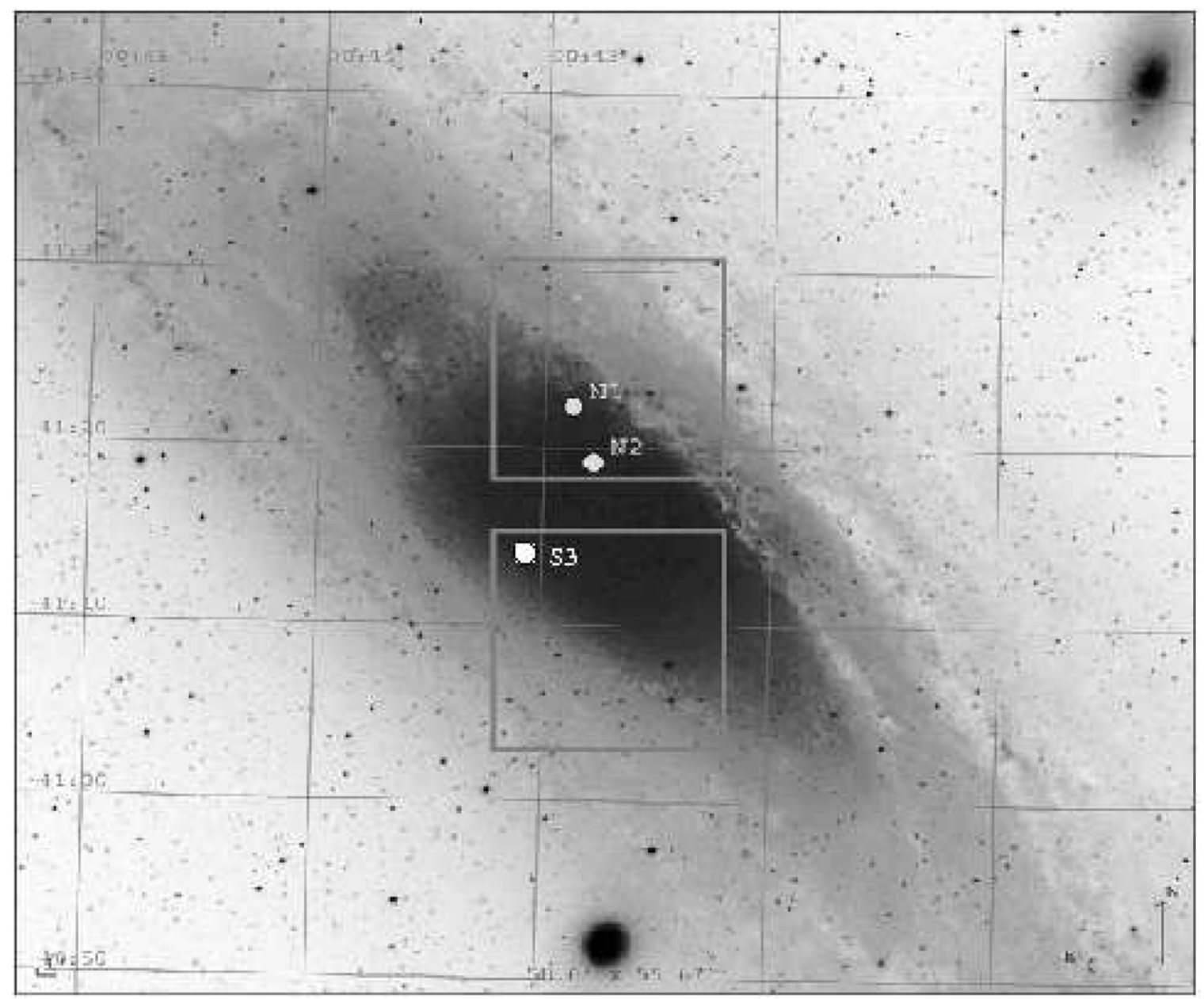

Figure 1. We display the fields of view of the OAB 2007-2010 PLAN pixel lensing campaign and the positions of our three microlensing candidates: OAB-07-N1, OAB-07-N2 (Calchi Novati et al. 2009a), and OAB-10-S3, the last of which first appeared in Lee et al. (2012) and is known as PAnd-4. The background is an archival image of M31.

Table 1

Observational Sampling for the 2006-2010 OAB Campaign

\begin{tabular}{lcccc}
\hline \hline$(1)$ & $(2)$ & $(3)$ & $(4)$ & $(5)$ \\
\hline 2006 & $1-11 \mathrm{Sep}$ & 11 & 8 & 4.2 \\
\hline 2007 & 12 Nov-31 Dec & $50^{\mathrm{a}}$ & 31 & 3.8 \\
2008 & 15 Sep-23 Nov & $65^{\mathrm{b}}$ & 38 & 4.6 \\
2009 & 17 Sep-22 Oct & 36 & 25 & 5.5 \\
2010 & 20 Sep-31 Oct & $41^{\mathrm{c}}$ & 20 & 4.6 \\
\hline Total & & 192 & 114 & 4.6 \\
\hline
\end{tabular}

Notes. The First-year Pilot Season is Then no Longer Considered within the Analysis. (1) Year; (2) period of the year; (3) number of nights awarded for our project; (4) number of nights with at least some M31 observations; (5) average number of hours/night of M31 observations. In the calculation of the last row, the data from the 2006 pilot season are not included. The PI for the 2006 and 2007 (2008-2010) proposals was F. Strafella (S. Calchi Novati). In 2010, we also took data at the $2 \mathrm{~m} \mathrm{HCT}$ telescope for 10 consecutive days, 1-10 October (PI A. Subramaniam).

${ }^{a} 12$ consecutive nights have been partly shared with another observer.

${ }^{b}$ Nine non-consecutive nights were partly shared with other observers and five full non-consecutive nights were allocated to other observers.

${ }^{c}$ One full night was allocated to another observer. "good" nights over the 192 allocated ones. Considering, however, the number of hours we could actually spend observing M31, with an average number of visibility hours given the period of the year and the declination of the site (44.4 north, almost ideal for observations toward M31) up to almost $10 \mathrm{hr} / \mathrm{night}$, the overall fraction of hours we could monitor M31 with respect to the allocated hourd drops to below $40 \%$. Although the quality of the data turned out to be good enough, we still had to reject a sizeable fraction of "bad" images (very poor seeing conditions and/or too high moon level). Specifically, within the selection pipeline, we do mask data points with large relative error bars; this further reduced the number of available data points to about 80-90 and 70-80, depending on the light curves, for $R$ - and $I$-band data, respectively. This number must be compared with the initial number of awarded nights, 192, for a fraction below $50 \%$. The sky brightness, because of anthropic pollution of the nearby towns, is about 1 mag brighter than in a typical dark site. The typical seeing values were around 2 " with a strong scatter, though, which further complicated the analysis.

In 2010, we submitted a proposal to carry out parallel observations to those at $\mathrm{OAB}$ at the $\mathrm{HCT}$ at the Indian Astronomical Observatory (http://www.iiap.res.in/iao/cycle.html) at $32^{\circ}$ north and located $4500 \mathrm{~m}$ above sea level, PI A. Subramaniam, and we were awarded 10 consecutive nights, October 1-10 (therefore, within the shift allocated at OAB) for $2 \mathrm{hr} /$ night. The photometric monitoring was carried out 
using the Himalayan Faint Object Spectrograph and Camera equipped with a Thompson CCD with read out noise $4.8 \mathrm{e}^{-}$ pixel $^{-1}$, gain 1.22 $\mathrm{ADU}_{\text {pixel }}{ }^{-1}$, pixel scale of $0^{\prime \prime} .296$ pixel $^{-1}$, and with $2048 \times 2048$ pixels for a total field of view of about $10^{\prime} \times$ $10^{\prime}$, slightly smaller than that at OAB. To match the OAB observations, we have observed two fields, north and south of the M31 center, centered at R.A. $=0^{\mathrm{h}} 42^{\mathrm{m}} 50^{\mathrm{s}}$ and decl. $=41^{\circ} 22^{\prime} 57^{\prime \prime}$ and decl. $=41^{\circ} 09^{\prime} 23^{\prime \prime}$, respectively, so as to be fully included within the OAB fields of view, with observations evenly distributed in two broadband filters, $R_{\mathrm{C}}$ and $I$. We obtained useful data out of all the 10 scheduled nights, with only some problems of guiding that forced us to reduce the exposure time down to 5 minutes/exposure against the programmed one of 10 minutes/ exposure.

\subsection{Data Analysis}

The raw data are first reduced with bias and (sky) flat field frames (plus defringing for $I$-band data) using the ccdred tasks within IRAF. The photometry is carried out according to the "superpixel" scheme, first introduced within the AGAPE group (Ansari et al. 1997) and further discussed in Calchi Novati et al. (2002, 2009a), which is fixed-size aperture photometry (we use $5 \times 5$ superpixels) with a linear empirical correction to account for the seeing variations. Several images (up to about 20) are taken each night per band and per field. The superpixel light curve is built with a weighted (by the inverse of the square of the flux error) average carried out after the seeing correction so as to end up with one data point per night per filter. This procedure is suitable to match the expected typical event duration of about a few days.

For the analysis of HCT data, right after the standard CCD reduction, we resample them so as to match the OAB pixel scale. For this purpose, first we draw a list of about 300 reference stars per field that we use to establish the relative astrometry and then, using the immatch tasks within IRAF, we carry out the pixel resampling (moving from the HCT 0 '.30 to the OAB 0 '.58 pixel scale). The rms of the relative astrometry on the resampled HCT images versus the $\mathrm{OAB}$ ones is at most 0.3 pixel. The resampled $\mathrm{HCT}$ data are then processed exactly as the OAB data.

\subsection{Pixel Lensing Pipeline}

The purpose of the pipeline is to establish a list of bona fide microlensing candidates. Within this scheme, our specific aim is to build a fully automated pipeline. This is crucial to deal with large data set, however the key aspect is that this enables us to reliably estimate the detection efficiency.

The pipeline we use closely follows that described in Calchi Novati et al. (2009a), which we refer the reader to for full details. Hereafter, we highlight the relevant steps. We work in the pixellensing regime so that the search for flux variations is carried out along all the pixels of the image. The analysis is carried out on OAB data working on each year separately. First, we have to establish a list of flux variations. To this end, we select light curves showing at least three consecutive points (one per night) above the baseline level at a $3 \sigma$ level, in both the $R$ and $I$ bands and then we ask for the threshold cut: for $R$-band data, $Q>50$, where $Q$ is the ratio of the $\chi^{2}$ of a straight line over a Paczyński fit (Calchi Novati et al. 2002, 2003). We recall that each flux variation is spread over a full cluster of nearby pixels whose identification therefore requires an analysis based on the spatial information of the images (Calchi Novati et al. 2002, 2009a). This way, we select a first sample of some 11,204 flux variations. As a second step, we want to remove all spurious variations (bad pixels, cosmic rays, variations induced by the seeing, and so on). To this end, we study the shape of the point-spread function (PSF) of the bump on a difference image obtained selecting images at the peak and images along the baseline having similar seeing conditions (Calchi Novati et al. 2009a). This way, the number of selected flux variations drops to 1827 . Next, we test the stability of the baseline, as indeed we expect most of these variations to be intrinsic variable signals. For this purpose, we carry out a Lomb-Scargle periodogram analysis (Lomb 1976; Scargle 1982) that we implement following Press et al. (1992) along three years of Isaac Newton Telescope (INT) data (Aurière et al. 2001; Paulin-Henriksson et al. 2003) and consider as a statistic the associated power $P$. As a threshold value to distinguish between noise and signal, we use $P=15$ (Figure 2). (As a test that our initial set of 11,204 variations is indeed dominated by spurious signals, therefore with most chances to show a stable baseline along the corresponding INT light curve, we find that most of the variations excluded with the PSF analysis have in fact $P<15$.) Experience (and superpixel photometry) teach us however that this way we may lose bona fide microlensing candidates whose light curves may be superimposed on a (possibly nearby) variable (as in the POINT-AGAPE PA-N1 event; Aurière et al. 2001). We therefore allow for flux variations with a variable baseline provided that the flux difference in the OAB data is significantly larger than the corresponding one in the INT data. The set of flux variations then reduces to 612. Finally, we adopt three further selection criteria to constrain the shape of the light curve: the first one for good enough sampling along the flux variations (Calchi Novati et al. 2009a), the second for compatibility with Paczyński, testing the reduced $\chi^{2}$ and asking $\chi^{2}<10$, and the last one for large enough variations, with a threshold on the flux difference at maximum magnification expressed in term of magnitude $\Delta R_{\max }<21.5$. This way, we are left with four microlensing candidate events: OAB-07-N1 and OAB-07-N2 already selected and presented in Calchi Novati et al. (2009a), with the second further discussed in Calchi Novati et al. (2010); a candidate from the 2008 data, further discussed below and finally eliminated from the selection; and, from the 2010 data, a microlensing candidate already reported by PAndromeda, PAnd-4 (Lee et al. 2012), that we also call OAB-10-S3 (N and S stand for north and south, respectively, the OAB field where the candidate is located).

The 2008 selected flux variation, in $\alpha, \delta=0^{\mathrm{h}} 42^{\mathrm{m}} 49^{\mathrm{s}} .22$, $41^{\circ} 22^{\prime} 42^{\prime \prime} .5(\mathrm{~J} 2000.0)$ at a distance of 6.6 from the M31 center, with maximum magnification at 4734 (JD-2450000.0), has a very short, half width at half maximum duration, $t_{\mathrm{FWHM}}$, below 3 days, and a quite bright bump, with flux difference at maximum magnification expressed in term of magnitude $\Delta R_{\max } \sim 20.0$ and color $R-I \sim 0.9$ (at the observed peak); Figure 3. On the other hand, the corresponding extension along the INT light curve shows a clear variable signal (with $P=31$ ). Indeed, the OAB data (although penalized by a shorter baseline per year of data), in 2008, as well as along the full four years of data, show evidence of that variation. A closer astrometric inspection, with an rms of the relative OAB-INT astrometry below the $0.2 \mathrm{OAB}$ pixel level, reveals that the INT variable sits some 4 INT pixels away from the pixel corresponding to the $\mathrm{OAB}$ variations, at a position that coincides with that of the variable identified also in the $\mathrm{OAB}$ data, $2 \mathrm{OAB}$ pixels away from that of the candidate (OAB and INT pixels cover 0.33 and 0.58 , respectively, for a distance of the candidate from the underlying variable of about $1^{\prime \prime} .2$ ). The selected flux variation is definitely at a different position with respect to the 

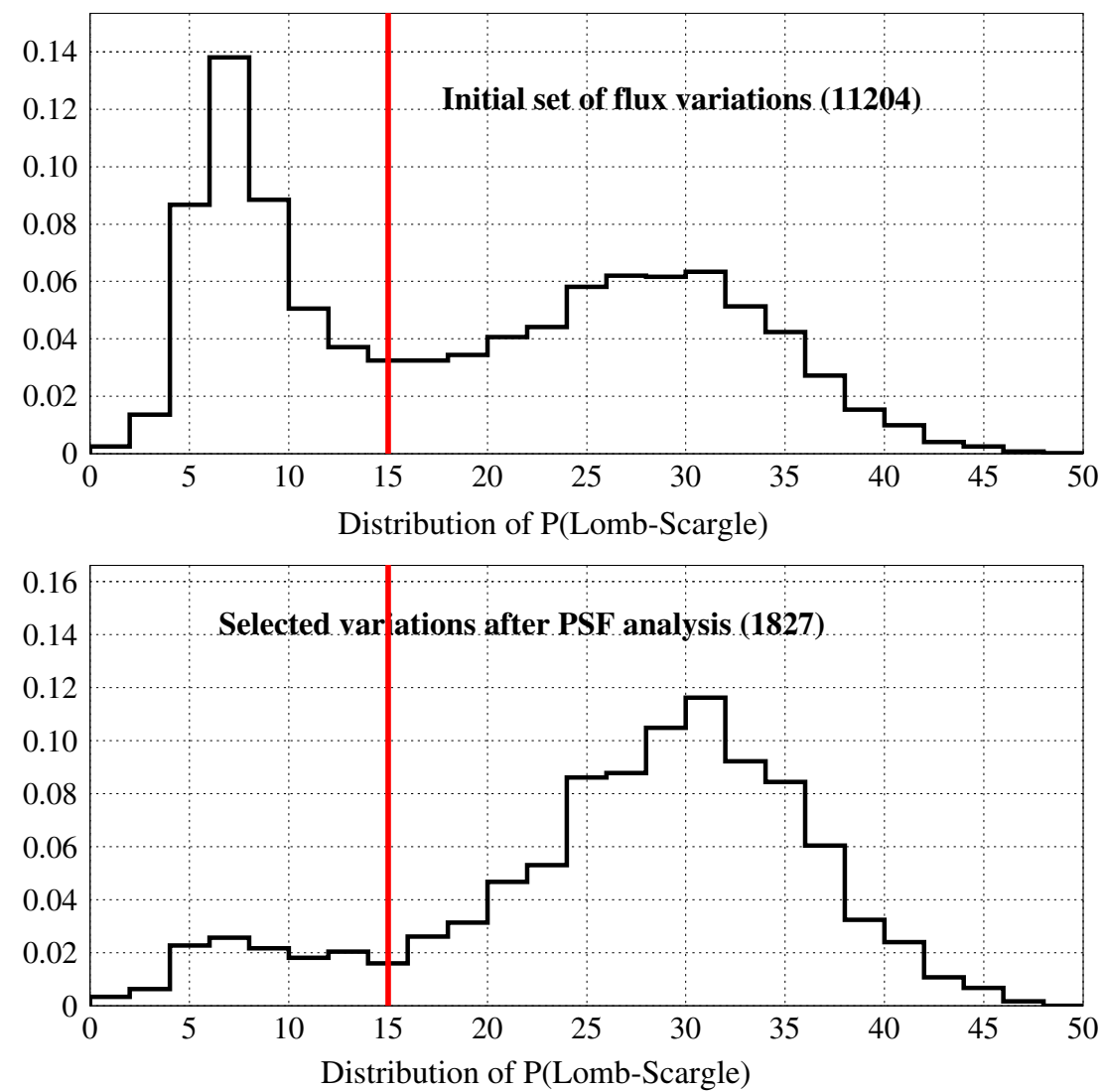

Figure 2. Normalized distribution for the "power," $P$, of the Lomb-Scargle periodogram analysis on the extension of our selected flux variations on the corresponding INT light curves. Top panel: the full set of initial selected light curves. Bottom panel: the surviving set of selected light curves after the PSF analysis (see the text for details). The vertical solid line at $P=15$ indicates the threshold value used within our selection pipeline.

(A color version of this figure is available in the online journal.)

underlying variable running along the same superpixel light curve of the microlensing candidate. From INT data, we infer the color of the variable as $R-I=1.1$, somewhat redder than the OAB variation, with peak magnitude $R=21.2$, more than 1 magnitude fainter than the $\mathrm{OAB}$ variation. As apparent from inspection of the OAB light curve, the sampling along the bump is poor, with a single data point (in both $R$ and $I$ data, with five images of that night per filter all clearly showing the variation and with no indications of any trend during the night) well above the variable baseline and no data, because of bad weather, on the three nights immediately before and after the peak. Additionally, a comparison of the two OAB light curves, one centered on the candidate and one centered on the position of the variable, strongly suggest that the flux excess with respect to the baseline for the data points immediately before and after the peak, at the origin of the initial trigger of this flux variation within the selection pipeline, should be attributed to the underlying variable rather than to the candidate, which, therefore, is left with a single significant data point (per band) along the bump. As an initial threshold, we ask for three consecutive points in each band above the baseline level at a $3 \sigma$ level. We are accordingly bound to exclude this flux variation, which our available sampling does not enable us to properly characterize, from our selection.

The remaining three candidates, on the other hand, all show a stable INT extension light curve (Lomb-Scargle power $P<7$ for all three of them), as well as a flat baseline in the OAB data for the years not including the bump. They are further discussed in their respective discovery papers; here we report their main characteristics (Table 2) and show their light curves (Figure 4).
Table 2

Main Characteristics of the Three Candidate Microlensing Events Selected within the Fully Automated 2007-2010 OAB Selection Pipeline

\begin{tabular}{lccc}
\hline \hline & OAB-07-N1 & OAB-07-N2 & OAB-10-S3 \\
\hline$\alpha(\mathrm{J} 2000)$ & $0^{\mathrm{h}} 42^{\mathrm{m}} 56^{\mathrm{s}} .70$ & $0^{\mathrm{h}} 42^{\mathrm{m}} 50^{\mathrm{s}} .36$ & $0^{\mathrm{h}} 43^{\mathrm{m}} 11^{\mathrm{s}} .52$ \\
$\delta(\mathrm{J} 2000)$ & $41^{\circ} 22^{\prime} 49^{\prime \prime} .8$ & $41^{\circ} 18^{\prime} 40^{\prime \prime} .1$ & $41^{\circ} 13^{\prime} 20^{\prime \prime} .2$ \\
$\Delta_{\mathrm{M} 31}(\operatorname{arcmin})$ & 7.1 & 2.8 & 5.8 \\
\hline$t_{0}(\mathrm{JD}-2450000.0)$ & $4434.0 \pm 0.2$ & $4466.1 \pm 0.1$ & $5485.1 \pm 0.3$ \\
$t_{\mathrm{FWHM}}($ days $)$ & $7.7 \pm 0.7$ & $1.4 \pm 0.3$ & $15 . \pm 2$. \\
$\Delta R_{\max }$ & $21.1 \pm 0.1$ & $19.2 \pm 0.2$ & $20.6 \pm 0.1$ \\
$R-I$ & $1.2 \pm 0.1$ & $1.2 \pm 0.3$ & $0.8 \pm 0.1$ \\
\hline
\end{tabular}

Notes. $t_{\text {FWHM }}$ and $\Delta R_{\max }$ are the duration and the flux difference from the baseline level, respectively, expressed in terms of magnitude according to the fit scheme of Gould (1996). For OAB-07-N1 and OAB-07-N2, the results are slightly different, still compatible within the errors, from those reported in Calchi Novati et al. (2009a, 2010) because of the extended baseline. For OAB-07-N2, we report the results of the fit along the joint data sets of OAB plus WeCAPP. For OAB-10-S3, we include within the fit the data acquired during our 2010 HCT campaign. OAB-10-S3 has been first published as PAnd-4 in Lee et al. (2012) and we are unable to explain the rather large difference in some of the reported values with respect to those reported in their Tables 3 and 4.

For the 2010 season, we also have at our disposal the additional HCT data set, with a smaller field of view than the $\mathrm{OAB}$ one (recovering a fraction of the area of about 60\%) and sampled along a baseline of 10 consecutive days, about onethird of the overall baseline of the OAB 2010 season. PAnd-4/ OAB-10-S3 lies within the HCT field of view. However, the last 

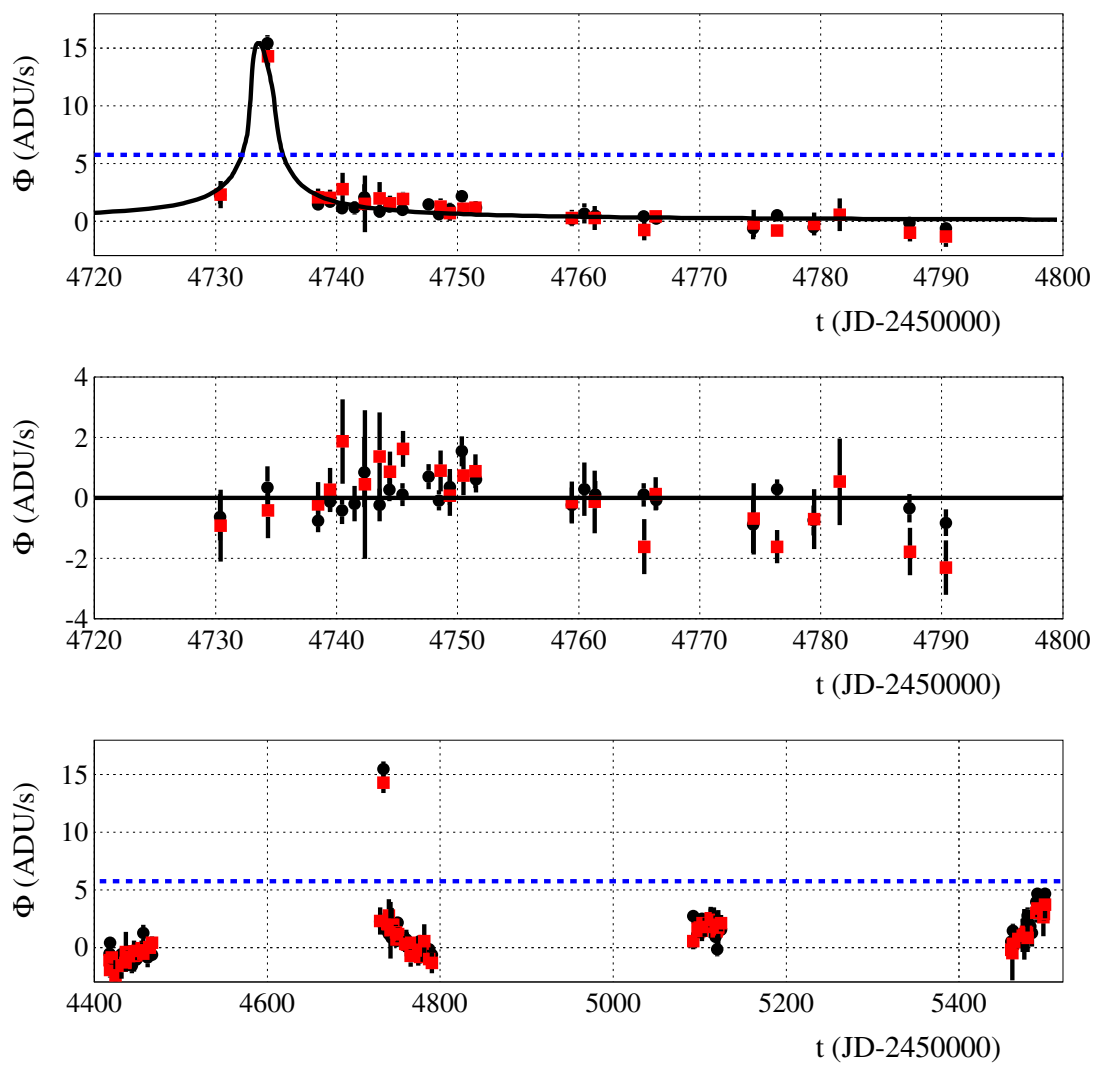

Figure 3. Flux variation detected in 2008 in $\mathrm{OAB}$ data selected by our automated selection pipeline but finally eliminated from the sample of microlensing candidates (see the text for details). Top panel: OAB 2008 light curve with the solid line representing the best Paczyński fit to the data. The dashed horizontal line indicates the flux difference with respect to the baseline corresponding to the flux deviation at maximum of the underlying variable as analyzed along the 1999-2001 INT data (see the text for details). Middle panel: OAB 2008 light curve of the residuals with respect to the best Paczyński fit. Bottom panel: OAB 2007-2010 light curve and dashed horizontal line as in the top panel. All panels: $R$ and $I$ band (rescaled) data are shown as circle and square symbols, respectively. The flux values on the $y$-axes are rescaled with respect to the $R$-band values.

(A color version of this figure is available in the online journal.)

HCT data point falls four nights before the peak of the event. However, as the event is rather long, the HCT data are still useful as they cover (and nicely overlap with the OAB data; Figure 4, bottom panel) the rising part and help us to better constrain the event lensing parameters.

As an additional analysis, we search for X-ray counterparts of our candidates using archival data. A positive match with a known X-ray source may indeed be an hint of a possible nonmicrolensing origin of the corresponding optical flux variation. In particular, we cross match our data with the M31 Deep XMMNewton Survey X-Ray Source Catalog (Stiele et al. 2011), an updated version with respect to what we used in our previous analysis (Calchi Novati et al. 2009a), where XMM refers to the X-Ray Multi-Mirror Mission. We also cross match with the Chandra analysis LMXBs in the bulge of M31 (Voss \& Gilfanov 2007). As for the astrometric precision, Stiele et al. (2011) report a $3 \sigma$ positional error for every entry, statistical and systematic, usually around a few arcsec; for the Chandra analysis, Voss $\&$ Gilfanov (2007) report an indicative range of values, from 0 .' 1 to $0 . .4$, depending on the brightness of the sources; finally, our astrometric solution is done using about 360 bright stars per field cross identified with sources in Massey et al. (2006) with a (statistical) rms below 0 !3. The nearest X-ray source to one of our candidates is that lying $25^{\prime \prime}$ from OAB-07-N2 (in the Chandra catalog, the nearest in the XMM-Newton one is reported at a distance of 27 ".2 with a positional error of 1 1.'84). For the given errors, we can safely rule out an identification. The same conclusion applies, a fortiori, for both OAB-07-N1 and OAB$10-\mathrm{S} 3$. For the first, the nearest source is found more than $1^{\prime}$ away (both catalogs); the second is at 35". 1 (Chandra) and 44". 4 with a positional error of 1 ". 83 (XMM-Newton). The situation is less clear for the 2008 flux variation, which we have eliminated from our analysis. A positive cross match of the positions looks plausible if we consider the XMM-Newton data, with a source at 5.9 with a positional error of 4.75 . This does not hold any more, however, both because of the smaller positional error and the increased distance to our flux variation ( $\left.7^{\prime \prime} .5\right)$, when we look at the Chandra data.

Finally, we may wonder, faced with the large possible variety of microlensing signals (binary lenses and so on), about the impact on our analysis of the requirement of compatibility with a Paczyński shape. If we drop this requirement, we end up with only two additional selected flux variations. The first is a clear variable signal, for which we have no available INT data but whose nature is revealed by the analysis of the light curve in the OAB data along the years not including the bump. The second is a more interesting case: a rather blue, $R-I \sim 0.3$, extremely bright, $\Delta R_{\max } \sim 17.6$, variation, located at $\alpha, \delta=0^{\mathrm{h}} 43^{\mathrm{m}} 02^{\mathrm{s}} .44,41^{\circ} 14^{\prime} 10^{\prime} .3(\mathrm{~J} 2000.0), 3^{\prime} .9$ from the M31 center, and occurring in 2008 around 4760 (JD-2450000.0) showing several peaks along a time scale of a few days as well as signs of chromaticity. We tried a binary lens fit (Bozza 2010) on this flux variation but we could not find any viable solution. Therefore, we attribute this flux variation to some 

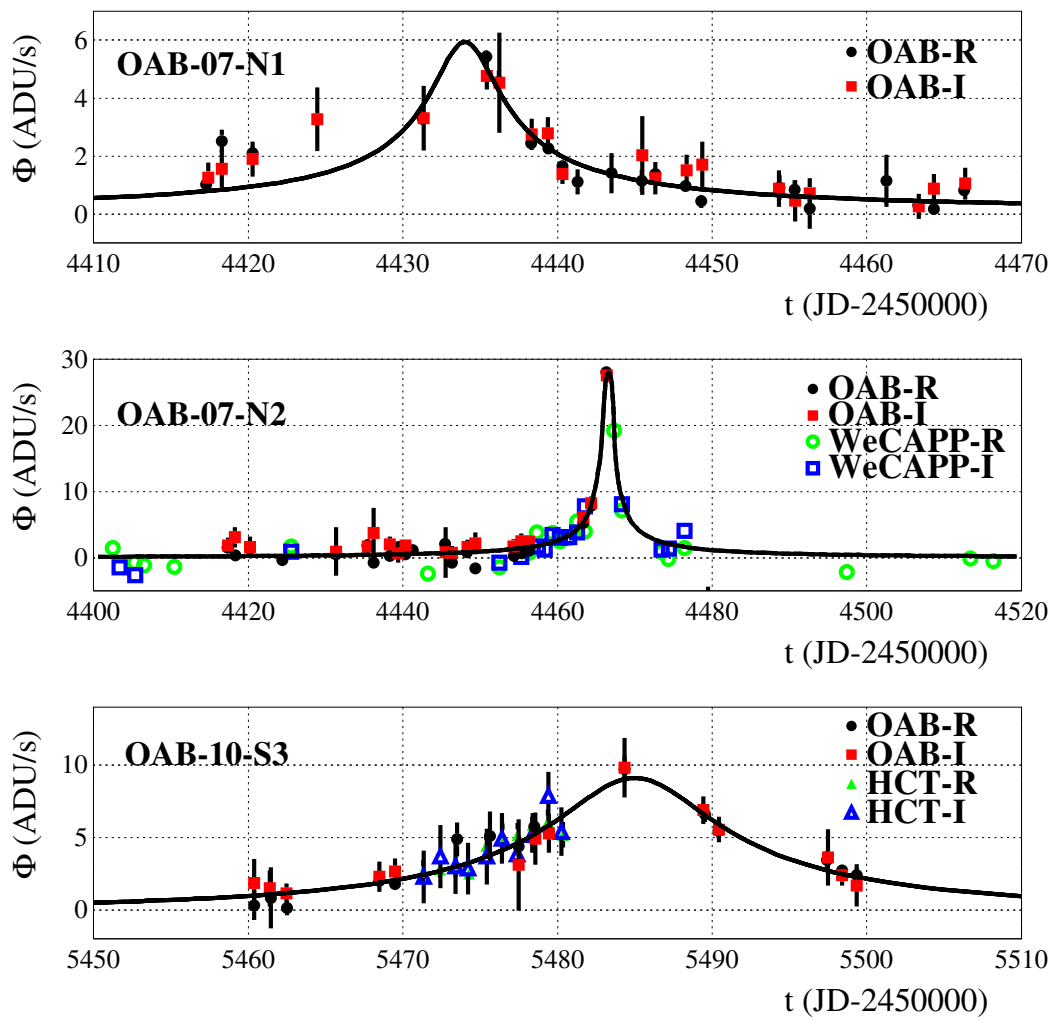

Figure 4. Light curves for the three selected microlensing events of our automated selection pipeline. From top to bottom: OAB-07-N1 and OAB-07-N2, both first presented in Calchi Novati et al. (2009a), and OAB-10-S3, first published as PAnd-4 in Lee et al. (2012). The solid line represents the best Paczyński fit to the data. Middle and bottom panels: besides the OAB data (filled symbols), we report the additional WeCAPP data also used for the analysis carried out in Calchi Novati et al. (2010); the additional HCT data of our 2010 campaign. The flux values on the $y$-axes are rescaled with respect to the OAB $R$-band values expressed in ADU s ${ }^{-1}$.

(A color version of this figure is available in the online journal.)

kind of unidentified cataclysmic variable. As above, we have checked for possible X-ray counterparts. The nearest (bright) source, from both the XMM-Newton and the Chandra catalogs, lies at about $25^{\prime \prime}$ and should therefore, for the given errors, be unrelated to this flux variation.

Lee et al. (2012) presented the results of the 2010 season of their PAndromeda (M31) pixel lensing survey. They used a $1.8 \mathrm{~m}$ telescope with a very wide field-of-view instrument $\left(7 \mathrm{deg}^{2}\right)$ and obtained 91 nights of data over about 5 months in two broadband ( $r_{\mathrm{P} 1}$ and $\left.i_{\mathrm{P} 1}\right)$ filters. In particular, they presented results for a search of microlensing events within the inner $40^{\prime} \times 40^{\prime}$ of M31. Overall, they reported six microlensing event candidates. We can use the 2010 PAndromeda results to test our OAB 2010 pipeline. In spite of the very large ratio of our field of view and the PAndromeda monitored field of view (about $20 \%$ considering only the (small) fraction of the overall field of view on which Lee et al. (2012) carried out their microlensing search, as a consequence of the sharp decrease of the expected signal moving outward from the M31 center), four out of the six PAndromeda candidates fall within the OAB fields of view (PAnd-1, 2, 3, and 4). Because of the much longer 2010 PAndromeda baseline, however, only two of these have been detected in October while the OAB campaign was going on (PAnd-1 and 4). As discussed, we find PAnd-4 to coincide with OAB-10-S3 also detected within our pipeline. PAnd-1, which has a very short duration, $t_{\mathrm{FWHM}}=3.1 \mathrm{~d}$ (Lee et al. 2012), unfortunately falls within a gap of the OAB sampling. On the OAB data, along its (short) bump, we detect two points, well above the $3 \sigma$ level of the baseline, according to our selection. However, this detection is clearly insufficient to characterize, if not even to trigger, a detection. The HCT data span exactly the moment of the PAnd-1 peak, unfortunately, however, PAnd-1 is not included within the HCT field of view. We may therefore conclude that the output of our pipeline and the PAndromeda pipeline are compatible. We consider this conclusion to also strengthen the results of the OAB pipeline for the previous years.

Single-bump, achromatic, suitably sampled with large enough $\mathrm{S} / \mathrm{N}$ Paczyński-like flux variations can be considered reliable microlensing candidates. Excluding binary lenses and/or similar cases where the intrinsic microlensing nature of the event can be accepted beyond any doubt, these flux variations are bound to remain within this limbo. A still possible background is that of cataclysmic variables, which are usually single-bump flux variations (at least within the time scale of the usual considered duration for the analysis of the baseline stability). However, these are usually bluer than the typical M31 microlensing candidates and, in particular to those discussed here, tend to show, as is typical for intrinsic variables, an asymmetry along the flux variation with a sharper rising part. For the case under examination, the intrinsic microlensing nature of two of the reported flux variations is further supported by additional data by WeCAPP, for OAB-07-N2 and HCT (presented here) and PAndromeda for OAB-10-S3. Indeed, the simultaneous detection on multiple pipelines and/or multiple data sets of the same flux variation, even if by itself cannot be taken as proof of the genuine microlensing nature of the flux variation, may make us more confident on its interpretation. This is for two main reasons. First, the joint analysis with additional data may further constrain the microlensing parameter space. Second, each 
pipeline (a fortiori with a different data set), in its broadest sense (data reduction, photometric analysis, flux variation search, and characterization), comes with its own systematics, which tend to be ruled out by multiple detections. More specifically, in Calchi Novati et al. (2009a) with OAB data alone, OAB-07-N2 was not fully sampled and we could only put forth a guess as to its microlensing nature. The joint analysis with the additional WeCAPP data then enabled us (Calchi Novati et al. 2010) to probe the symmetric and achromatic shape of the full flux variation then confirmed the microlensing interpretation. Furthermore, the dense sampling made possible a much more refined analysis of the microlensing parameter space. Indeed, together with an additional analysis of the underlying source flux from archival data, the joint OAB plus WeCAPP light curve enabled us to conclude, even if only marginally, through a study of the lens proper motion (Gould 1994; Han \& Gould 1996), in favor of the MACHO nature of the lens. As for OAB-10-S3, the HCT data presented here, even if not necessary to enhance its detection, enabled us to better characterize the microlensing parameters. This flux variation is the selected as a microlensing candidate by two completely independent pipelines (on different data sets), specifically, besides the present one, also by PAndromeda as PAnd-4 (Lee et al. 2012). For purposes of the following analysis, we therefore will consider the three flux variations selected within our pipeline as bona fide microlensing variations.

\subsection{Monte Carlo and Efficiency Analyses}

For the analysis of the expected signal, we closely follow the scheme outlined in Calchi Novati et al. (2009a) and references therein, to which we refer the reader for full details. First, we build a Monte Carlo simulation based on the original work in Baillon et al. (1993); Ansari et al. (1997) where, in addition to the astrophysical model of all the quantities of interest, we simulate the microlensing flux variations. The evaluation of the expected signal for a microlensing experiment is based on the microlensing rate (Griest 1991; Calchi Novati et al. 2008), with the specific case of M31 pixel lensing also discussed in Han (1996), Baltz \& Silk (2000), Gyuk \& Crotts (2000), Kerins et al. (2001), and Riffeser et al. (2006). Our model of M31 is based on the Kent (1989) data. For both the M31 bulge and disk stars, we make use of a synthetic luminosity function extracted from an Instituto de Astrofísica de Canarias star (Aparicio \& Gallart 2004) with sources expected up to a magnitude of roughly $M_{I} \sim 2$. Finally, the flux variations are simulated as single-lens microlensing events accounting for finite source size (Witt \& Mao 1994) and we reproduce the observational conditions, in particular the sampling, of our OAB campaign. Within the Monte Carlo simulation, we carry out a first (knowingly over-optimistic) selection pipeline asking for the flux variations to have at least three consecutive points $3 \sigma$ above the baseline level. Monte-Carlo-selected light curves may, however, not be selected within our data set. Within the Monte Carlo simulation, we cannot in particular reproduce those steps of our pipeline where the spatial information across the images comes into play: the cluster analysis we carry out to identify the initial set of flux variations and the PSF analysis we use to exclude spurious signals; additionally, within the Monte Carlo simulation, we do not reproduce all the problems intrinsic to the images such as crowding, background flux variations due to underlying variables, and so on; all these aspects must, however, be taken into account. To get to a reliable estimate of the expected signal, we therefore inject, making use of the daophot tasks within IRAF, (part) of these Monte Carlo- selected light curves on the real data ( $R$ band only), just after the basic CCD reduction, and then run our selection pipeline from scratch. Finally, as a result, we end up with the distributions of the parameters and the number of events for the expected signal. As lens populations, we consider M31 bulge and lens stars ("self-lensing") and MACHO lensing as MACHOs belonging to the M31 and MW halos, for which we study a set of delta mass functions within the range $10^{-3}-1 M_{\odot}$. Specifically, to minimize the statistical noise on the number of expected events, within the Monte Carlo simulation we simulate $10^{9}$ events for self lensing and as much for each mass of MACHO lensing, per year. Out of the selected events within the Monte Carlo simulation, we then inject 12,000 events for self lensing and for each MACHO mass value per year (to avoid overlaps among the injected events on the images we split the analysis so as to have 500 events per field for each run).

\subsection{The Statistics on MACHOs}

The driving astrophysical question of the present analysis is the MACHO content in galactic halos. It is therefore of primary interest to address the issue of the nature of the observed events, whether self lensing or MACHO. Besides the previouslydiscussed and peculiar case of OAB-07-N2, the main statistics at our disposal, also considering the power of investigation within the detection efficiency analysis, are the magnitude at maximum and the color, which at most can be used to assess the coherence of the analysis with the expected signal with no reference, however, to the specific lens population, the duration, and the position. As for the duration, $t_{\mathrm{FWHM}}$, we recall from previous analyses (Kerins et al. 2001; Calchi Novati et al. 2005; Riffeser et al. 2006) and specifically for the OAB data, our analysis in Calchi Novati et al. (2009a) that most events are expected, as indeed we find, to last fewer than about 10-20 days. However, this parameter is unfit to distinguish among the lens populations, at least for those of roughly equal lens mass (thus, specifically, for stellar lenses and MACHOs around $0.5 M_{\odot}$ ) for which one would rather need a reliable estimate of the Einstein time. On the other hand, the small number of events at our disposal makes the position of the events of limited use to this purpose, moreover it should be recalled that the analysis of the originally proposed signature of M31 MACHO lensing versus self lensing due to the M31 disk inclination may in fact be complicated by effects of differential extinction (An et al. 2004; Montalto et al. 2009). Still, self-lensing events tend to be more clustered than MACHOs around the M31 center and specifically this holds for our experimental configuration (Calchi Novati et al. 2009a). In fact, this is the underlying motivation, in the following likelihood analysis, for binning the space in units of distance from the M31 center (Figure 5, top panel). The main statistics to disentangle MACHO and self-lensing signals that are left are therefore the expected number of events. The results of our analysis, Monte Carlo simulation followed by the efficiency analysis, are as follows. For self lensing, we expect $2.2 \pm 0.2$ events. For MACHO lensing, for full $(f=1) \mathrm{M} 31$ and MW halos, we expect from about four up to above seven events, according to the mass value (Figure 5, middle panel), with a statistical relative error around $10 \%$, with a maximum for the expected signal at $10^{-2} M_{\odot}$ before a decrease, for $10^{-3} M_{\odot}$, due to the drop of sensitivity for very short duration events related to our (insufficient) sampling. The reported statistical error is that associated with the Monte Carlo simulation, for which the (Poisson) error scales as the square root of the simulated events (Section 5.1 of Calchi Novati et al. 2005), with the final budget 

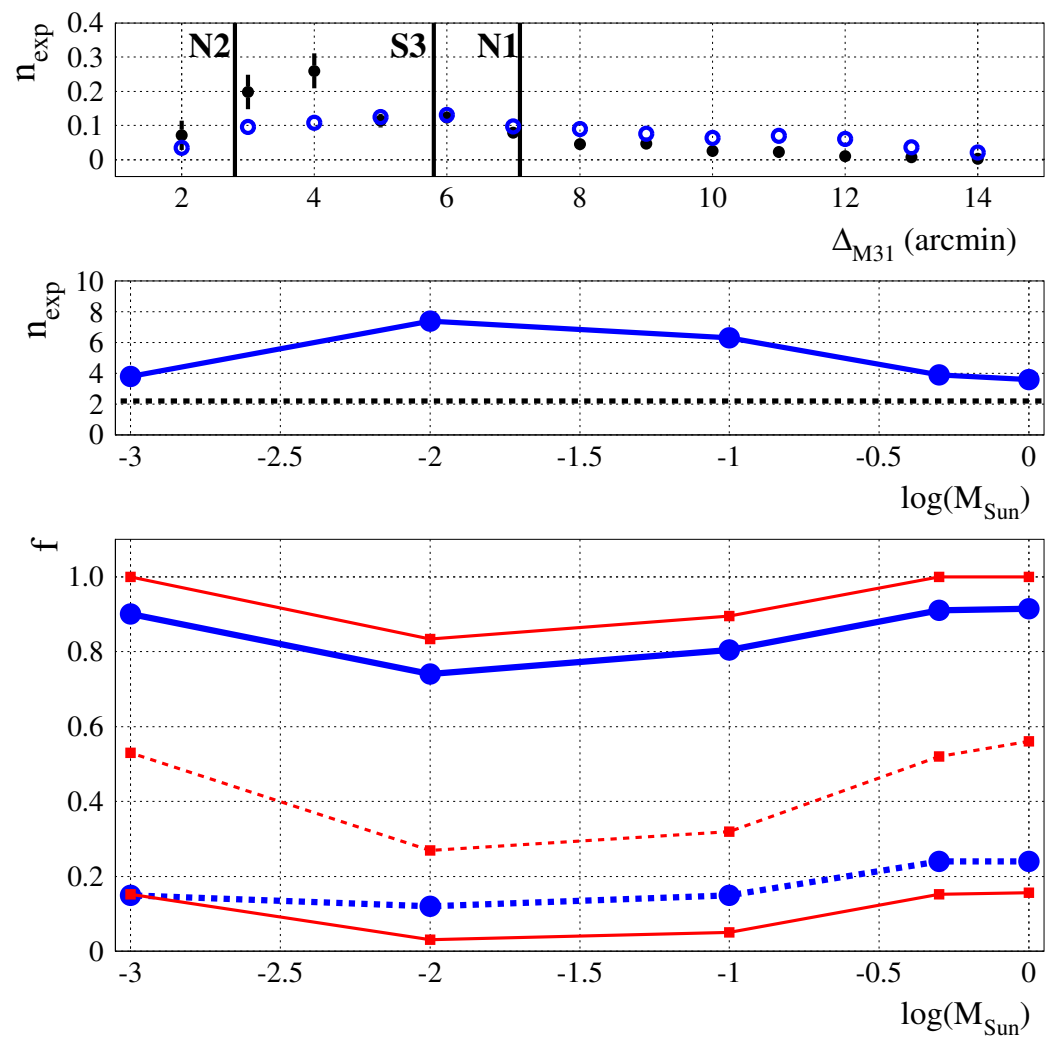

Figure 5. Top panel: the (normalized) expected number of events for self lensing (filled symbols) and MACHO lensing as a function of the distance to the M31 center (arcmin). The (statistical) error bars appear only when they exceed the size of the symbols. The solid vertical lines indicate the position within this parameter space of the selected candidates. Middle panel: the number of expected MACHO lensing events, for full (M31 and MW) halos $(f=1)$ as a function of the (logarithm of the) MACHO mass; the horizontal dashed line indicates the expected number of self-lensing events. Bottom panel: limits on the halo mass fraction in the form of MACHOs, $f$, as a function of the (logarithm of the) MACHO mass obtained from a Bayesian analysis based on the evaluation of the likelihood comparing the expected, MACHO, and self-lensing signals for the three observed events (see the text for details). Circle symbols: modal values (dashed lines) and upper 95\% CL limit in the case where no a priori hypotheses are made on the nature of the observed events. Square symbols (and thin lines): modal values (dashed lines) and upper and lower 95\% CL limits obtained under the additional hypothesis that, following the analysis in Calchi Novati et al. (2010), OAB-07-N2 is a MACHO lensing event.

(A color version of this figure is available in the online journal.)

dominated by large by the error on the efficiency. Next, we may compare this result for the expected signal with $n_{\mathrm{obs}}=3$ observed events with the additional information, as already recalled, that OAB-07-N2 may indeed be rather a MACHO event than a self-lensing event. Leaving aside this information for the moment, the conclusion of the analysis, based on the bare numbers, is that self lensing is fully able to explain the observed rate. Specifically, according to the Poisson statistics followed by the number of events, for the given expected mean signal (and excluding for the moment the MACHO signal), the 95\% CL upper limit is for 6.3 events, well above the observed rate. We have then to consider the expected MACHO lensing. Here, a fundamental remark is that the expected signal, for full halos, is not much larger than the self-lensing one. Together, these results suggest that the current experiment is unable to set lower limits on MACHOs and can establish, if any, only rather weak upper limits. On the other hand, that same smallness of the expected MACHO lensing signal renders, as soon as we acknowledge the MACHO nature of even only one event, as we may do for OAB-07-N2 according to the analysis in Calchi Novati et al. (2010), the situation altogether different as we may expect at once to find a sizeable lower limit on the halo mass fraction in the form of MACHOs.

We can quantify the above statements by inferring from the data a probability distribution for $f$ through a Bayesian analysis based first on the evaluation of the likelihood function. To this end, we closely follow the approach outlined in Calchi Novati et al. (2005; for a specific discussion on the use of the likelihood within a microlensing analysis, we also refer the reader to Calchi Novati et al. 2013). We bin the entire field of view into $N_{\text {bin }}$ bins; for each we have the model prediction $x_{i}\left(i=1, \ldots, N_{\text {bin }}\right)$ and the observed number of events $n_{i}$. For the fixed model, the different $x_{i}$ are not independent as they all depend on the halo fraction, $f$ :

$$
x_{i}(f, m)=b_{i}+f s_{i}(f=1, m),
$$

with $m$ being the MACHO mass and where the signal we look for, $s$, is the number of MACHO events. The background, $b$, is the self-lensing signal (as our purpose is to constrain $f$, we may rather refer to the signal as the product $f \cdot s$ ). The likelihood is the product of the individual probabilities of the $n_{i}$ in each bin:

$$
L\left(n_{i} \mid x_{i}\right)=\prod_{i=1}^{N_{\text {bin }}} \frac{1}{n_{i} !} e^{-x_{i}} x_{i}^{n_{i}}=e^{-N_{\text {exp }}} \prod_{i=1}^{N_{\text {obs }}} x_{i},
$$

where in the second step we have specialized the bins to contain either 0 or 1 observed event (Gould 2003). As previously addressed, for binning, we choose the distance from the M31 center and we use $1^{\prime}$ bins. Keeping the MACHO mass fixed as a parameter, for a flat prior for $f$ different from zero in the interval $(0,1)$, given the likelihood, we can then evaluate the 
probability distribution $P(f)$. We evaluate the modal value for $f$ and, around it, the $95 \%$ CL region that then defines the lower and the upper limits. The results of this analysis are shown in Figure 5, bottom panel. If we do not introduce any prior within the analysis, as anticipated, the observed signal turns out to be compatible with the expected self-lensing rate, with no lower limits for $f$ and upper limits above $70 \%$ (90\% in the range $\left.0.5-1 M_{\odot}\right)$. If instead we impose that OAB-07-N2 is a MACHO, namely within Equation (1) we set the self-lensing background to zero, $b=0$, and we find a sizeable (15\%) lower limit for $f$ in the mass range $0.5-1 M_{\odot}$, with no upper limit and somewhat smaller limits moving down to $10^{-2} M_{\odot}$. Finally, the observed rate ( 3 events) is in fact also compatible with no self lensing (for an expected signal of 2.2 events). If we assume this extreme case as a working hypothesis, the lower limit for $f$ in the mass range $0.5-1 M_{\odot}$ would rise to about $30 \%$.

\section{CONCLUSIONS}

We have presented the final analysis of the four-year (2007-2010) pixel lensing campaign of the PLAN collaboration toward M31 aimed at the search and the characterization of microlensing events. The driving scientific motivation is the search for a dark matter signal in galactic halos in form of compact objects, MACHOs. The specific aim of the campaign is to better understand the signal coming from the putative MACHO population as compared with the background signal of selflensing events, defined as opposed to MACHO lensing with the lens belonging to known stellar (M31) populations. To this end, we monitored the central region of M31, where the expected rate is larger for both signals, still with self lensing expected to be more clustered around the M31 center, looking for new microlensing events. A key aspect of our analysis is the use of a fully automated pipeline for the search of microlensing-like flux variations which, besides leading us to the determination of a set of microlensing candidates, enables us to reliably estimate the detection efficiency. For a given astrophysical model, this eventually enables us to reliably estimate the expected signal through a Monte Carlo simulation of the experiment.

The analysis is based on data collected at the $1.5 \mathrm{~m}$ telescope of the OAB in two broad $R$ and $I$ bands in two fields $13^{\prime} \times$ 12 .7 around the M31 center. After a first year pilot season (2006; Calchi Novati et al. 2007), the observational campaign eventually lasted four years (2007-2010) with an awarded baseline to our survey, on average, of some 48 night/year plus, in 2010, 10 consecutive nights of complementary data from the $2 \mathrm{~m} \mathrm{HCT}$. Altogether, however, bad weather and/or generally unsuitable observational conditions introduced several gaps within our sampling with the final analysis based on the data collected, overall, during about 90 nights (excluding the contribution of HCT data). The expected short duration of the microlensing flux variations, together with the small rate of events, magnifies the impact of this problem. Indeed, this is made explicit also from the analysis presented in this paper. We have discussed the case of a flux variation preliminarily selected, in the 2008 season, but finally rejected because of incomplete sampling along the bump. For the same reason, we did not select the microlensing candidate PAnd-1 (Lee et al. 2012) even if it was included within our field of view and baseline.

The results of the pipeline are as follows. Overall, we select three microlensing candidate events: OAB-07-N1 and OAB07-N2, both first presented in Calchi Novati et al. (2009a), and a third candidate that occurred during the 2010 season and has already been published by PAndromeda in Lee et al.
(2012) as PAnd-4, which we also call OAB-10-S3, and for which we also have data from HCT. The results of our pipeline for the 2010 season turn out to be compatible with those of PAndromeda (Lee et al. 2012; thus strengthening its conclusions also for the previous seasons). As discussed within the text, the detection of the same flux variation on multiple pipelines and/ or data sets is useful for the purpose of its interpretation as a microlensing candidate. First, additional data may help to better constrain the candidate microlensing parameter space. Second, any independent detection comes with the bonus of removing, if any, the systematics of each pipeline. Besides the case of OAB-10-S3 (PAnd-4), we recall OAB-07-N2, first presented in Calchi Novati et al. (2009a), with which we could perform a new analysis thanks to additional WeCAPP data (Calchi Novati et al. 2010). In particular, these data made possible a refined analysis of the lensing parameter space, specifically of the lens proper motion, which enabled us to conclude in favor of the MACHO nature of the lens. For purposes of the analysis, we consider all three candidates as bona fide microlensing variations.

The observed rate, based on the number of events, is compatible with the expected self-lensing signal. A major outcome of our analysis, though, is that the expected MACHO lensing, for the full M31 and MW halos, is only marginally larger than self lensing, which is a different situation from analyses toward the Magellanic Clouds, where the expected self-lensing signal is evaluated to be much smaller than that of MACHO lensing. This result, together with the small statistics of the events at our disposal, prevents us from putting strong constraints on the putative MACHO population. This situation makes extremely important, whenever possible, the detailed analysis of single events addressing the issue of their nature, as was the case for the event POINT-AGAPE-S3/WeCAPP-GL1 (Riffeser et al. 2008), and as we could do for OAB-07-N2 (Calchi Novati et al. 2010). Indeed, the hypothesis of the MACHO nature of OAB-07-N2, as suggested by that last analysis, drives a sizeable lower limit on the halo mass fraction in the form of MACHOs, $f$. Quantitatively, we evaluate an expected self-lensing signal of 2.2 events, fully compatible therefore with our 3 observed events, and MACHO lensing, for full halos, of 4-7 events moving through our chosen range of masses $\left(10^{-3}-1 M_{\odot}\right)$. In particular, we evaluate an expected signal of 3.9 events for $0.5 M_{\odot}$ which, under the hypothesis that OAB-07-N2 is a MACHO, translates into a lower limit for $f$ of about $15 \%$. This outcome makes apparent the need of carrying out similar analyses using larger sets of events, possibly across larger fields of view, for which, besides their number, also additional statistics may be used to disentangle the MACHO signal from the self-lensing signal. From this perspective, the observational campaign PAndromeda (Lee et al. 2012) promises to mark an important further step in the understanding of this issue.

We thank M. Fitzpatrick for his support with IRAF. A.G. was supported by NSF grant AST-1103471. M.D. is thankful to Qatar National Research Fund (QNRF), member of Qatar Foundation, for support by grant NPRP 09-476-1-078. P.J. acknowledges support by the Swiss National Science Foundation.

\section{REFERENCES}

Alard, C. 2001, MNRAS, 320, 341

Alcock, C., Allsman, R. A., Alves, D. R., et al. 2000, ApJ, 542, 281 Alves, D. R., \& Nelson, C. A. 2000, ApJ, 542, 789

An, J. H., Evans, N. W., Hewett, P., et al. 2004, MNRAS, 351, 1071 Ansari, R., Auriere, M., Baillon, P., et al. 1997, A\&A, 324, 843 
Aparicio, A., \& Gallart, C. 2004, AJ, 128, 1465

Aubourg, É., Palanque-Delabrouille, N., Salati, P., Spiro, M., \& Taillet, R. 1999, A\&A, 347, 850

Aurière, M., Baillon, P., Bouquet, A., et al. 2001, ApJL, 553, L137

Baillon, P., Bouquet, A., Giraud-Heraud, Y., \& Kaplan, J. 1993, A\&A, 277, 1

Baltz, E. A., \& Silk, J. 2000, ApJ, 530, 578

Belokurov, V., An, J., Evans, N. W., et al. 2005, MNRAS, 357, 17

Bennett, D. P. 2005, ApJ, 633, 906

Besla, G., Hernquist, L., \& Loeb, A. 2013, MNRAS, 428, 2342

Bozza, V. 2010, MNRAS, 408, 2188

Calchi Novati, S. 2010, GReGr, 42, 2101 (Invited Review)

Calchi Novati, S., Bozza, V., De Paolis, F., et al. 2009a, ApJ, 695, 442

Calchi Novati, S., Covone, G., de Paolis, F., et al. 2007, A\&A, 469, 115

Calchi Novati, S., Dall'Ora, M., Gould, A., et al. 2010, ApJ, 717, 987

Calchi Novati, S., de Luca, F., Jetzer, P., Mancini, L., \& Scarpetta, G. 2008, A\&A, 480, 723

Calchi Novati, S., De Luca, F., Jetzer, P., \& Scarpetta, G. 2006, A\&A, 459, 407

Calchi Novati, S., Iovane, G., Marino, A. A., et al. 2002, A\&A, 381, 848

Calchi Novati, S., Jetzer, P., Scarpetta, G., et al. 2003, A\&A, 405, 851

Calchi Novati, S., \& Mancini, L. 2011, MNRAS, 416, 1292

Calchi Novati, S., Mancini, L., Scarpetta, G., \& Wyrzykowski, Ł. 2009b, MNRAS, 400, 1625

Calchi Novati, S., Mirzoyan, S., Jetzer, P., \& Scarpetta, G. 2013, MNRAS, 435,1582

Calchi Novati, S., Paulin-Henriksson, S., An, J., et al. 2005, A\&A, 443, 911

Crotts, A. P. S. 1992, ApJL, 399, L43

de Jong, J. T. A., Kuijken, K., Crotts, A. P. S., et al. 2004, A\&A, 417, 461

de Jong, J. T. A., Widrow, L. M., Cseresnjes, P., et al. 2006, A\&A, 446, 855

de Rujula, A., Giudice, G. F., Mollerach, S., \& Roulet, E. 1995, MNRAS, 275,545

Dominik, M. 2009, MNRAS, 393, 816

Evans, N. W., \& Kerins, E. 2000, ApJ, 529, 917

Gondolo, P. 1999, ApJL, 510, L29

Gould, A. 1994, ApJL, 421, L71

Gould, A. 1995, ApJ, 441, 77

Gould, A. 1996, ApJ, 470, 201

Gould, A. 1998, ApJ, 499, 728

Gould, A. 2003, ApJ, 583, 765

Griest, K. 1991, ApJ, 366, 412

Griest, K., \& Thomas, C. L. 2005, MNRAS, 359, 464

Gyuk, G., \& Crotts, A. 2000, ApJ, 535, 621

Gyuk, G., Dalal, N., \& Griest, K. 2000, ApJ, 535, 90

Han, C. 1996, ApJ, 472, 108
Han, C., \& Gould, A. 1996, ApJ, 467, 540

Ingrosso, G., Calchi Novati, S., de Paolis, F., et al. 2006, A\&A, 445, 375

Ingrosso, G., Calchi Novati, S., de Paolis, F., et al. 2007, A\&A, 462, 895

Jetzer, P. 1994, A\&A, 286, 426

Jetzer, P., Mancini, L., \& Scarpetta, G. 2002, A\&A, 393, 129

Kent, S. M. 1989, AJ, 97, 1614

Kerins, E., Carr, B. J., Evans, N. W., et al. 2001, MNRAS, 323, 13

Lee, C.-H., Riffeser, A., Koppenhoefer, J., et al. 2012, AJ, 143, 89

Lomb, N. R. 1976, Ap\&SS, 39, 447

Mancini, L., Calchi Novati, S., Jetzer, P., \& Scarpetta, G. 2004, A\&A, 427, 61

Massey, P., Olsen, K. A. G., Hodge, P. W., et al. 2006, AJ, 131, 2478

Moniez, M. 2010, GReGr, 42, 2047 (Invited Review)

Montalto, M., Seitz, S., Riffeser, A., et al. 2009, A\&A, 507, 283

Paczyński, B. 1986, ApJ, 304, 1

Paulin-Henriksson, S., Baillon, P., Bouquet, A., et al. 2003, A\&A, 405, 15

Press, W. H., Teukolsky, S. A., Vetterling, W. T., \& Flannery, B. P. 1992, Numerical Recipes in FORTRAN. The Art of Scientific Computing (2nd ed.; Cambridge: Cambridge Univ. Press)

Riffeser, A., Fliri, J., Bender, R., Seitz, S., \& Gössl, C. A. 2003, ApJL, 599, L17

Riffeser, A., Fliri, J., Gössl, C. A., et al. 2001, A\&A, 379, 362

Riffeser, A., Fliri, J., Seitz, S., \& Bender, R. 2006, ApJS, 163, 225

Riffeser, A., Seitz, S., \& Bender, R. 2008, ApJ, 684, 1093

Roulet, E., \& Mollerach, S. 1997, PhR, 279, 67

Sahu, K. C. 1994, Natur, 370, 275

Salati, P., Taillet, R., Aubourg, É., Palanque-Delabrouille, N., \& Spiro, M. 1999, A\&A, 350, L57

Scargle, J. D. 1982, ApJ, 263, 835

Stiele, H., Pietsch, W., Haberl, F., et al. 2011, A\&A, 534, A55

Strigari, L. E. 2013, PhR, 531, 1

Tisserand, P., Le Guillou, L., Afonso, C., et al. 2007, A\&A, 469, 387

Voss, R., \& Gilfanov, M. 2007, A\&A, 468, 49

Witt, H. J., \& Mao, S. 1994, ApJ, 430, 505

Wozniak, P., \& Paczynski, B. 1997, ApJ, 487, 55

Wu, X.-P. 1994, ApJ, 435, 66

Wyrzykowski, Ł., Kozłowski, S., Skowron, J., et al. 2009, MNRAS, 397, 1228

Wyrzykowski, Ł., Kozłowski, S., Skowron, J., et al. 2010, MNRAS, 407, 189

Wyrzykowski, Ł., Kozłowski, S., Skowron, J., et al. 2011a, MNRAS, 413, 493

Wyrzykowski, L., Skowron, J., Kozłowski, S., et al. 2011b, MNRAS, 416, 2949

Zaritsky, D. 2004, ApJL, 614, L37

Zaritsky, D., \& Lin, D. N. C. 1997, AJ, 114, 2545

Zhao, H., \& de Zeeuw, P. T. 1998, MNRAS, 297, 449 